\title{
A Monoclonal Antibody Recognizes Follicular Granulosa Cell Antigens in Porcine Ovaries
}

\author{
Chiemi TAJIMA ${ }^{1)}$, Noboru MANABE ${ }^{1)}$, Naoko INOUE'1), Toshikatsu MATSUI ${ }^{1)}$, \\ Noriko KAGAWA ${ }^{1)}$, Eiichi HONDO' ${ }^{2)}$, Takashi MIYANO ${ }^{3)}$, Jozsef RATKY4) and \\ Hajime MIYAMOTO ${ }^{1)}$
}

\author{
1) Unit of Anatomy and Cell Biology, Department of Animal Sciences, Kyoto University, Kyoto \\ 606-8502, ${ }^{2)}$ Department of Veterinary Anatomy, Faculty of Agriculture, Yamaguchi \\ University, Yamaguchi 753-8515, ${ }^{3)}$ Department of Animal Sciences, Kobe University, Kobe \\ 657-8501, Japan and ${ }^{4)}$ Research Institute for Animal Breeding and Nutrition, Herceghalom, \\ H-2053, Hungary
}

\begin{abstract}
We prepared an IgM monoclonal (PAG-1) antibody against follicular granulosa cells of porcine ovaries. Immunohistochemical reaction of the antibody was detected only in follicular granulosa cells, not in any other ovarian tissues or organs. Western blotting analysis demonstrated that $38-$ and $42-\mathrm{kD}$ bands were present in the granulosa cell membrane samples prepared from healthy follicles. In the samples from early atretic and progressed atretic follicles, 38- and 64-kD bands were shown. During follicular atresia, the $64-\mathrm{kD}$ band increased. After glycosidase treatment, the 64$\mathrm{kD}$ band shifted to $42-\mathrm{kD}$, but no change was seen in the 38- and 42-kD bands. The present results indicate that the $42-\mathrm{kD}$ band membrane protein is glucoconjugated during atresia, resulting in a glycoconjugated $64-\mathrm{kD}$ protein appearing in the granulosa cells of the atretic follicles. We hypothesize that the glycoconjugated cell membrane protein (64-kD glycoprotein) may act as a trigger for phagocytosis in neighboring granulosa cells in atretic follicles.
\end{abstract}

Key words: Apoptosis, Granulosa cell, Monoclonal antibody, Ovarian follicle, Pig

(J. Reprod. Dev. 48: 567-572, 2002)

I n mammalian ovaries, more than $99 \%$ of follicles undergo a degenerative change, called "atresia", at varying stages of follicular development [1]. Recent findings have suggested that the degeneration of atretic follicles in mammalian ovaries can be explained, at least in part, by apoptotic cell death of granulosa and internal theca cells $[2,3]$. In porcine ovaries, apoptosis demonstrated histochemically by the terminal deoxynucleotidyl transferase-mediated biotinylated deoxyuridine triphosphate nick endlabeling (TUNEL) method was seen in scattered granulosa cells located on the inner surface of the

Accepted for publication: June 12, 2002

Correspondence: N. Manabe follicular wall, but not in cumulus cells, internal or external theca cells, or oocytes in the early stage of atresia [4-6]. Electron microscopic observation showed that condensed nuclei, a typical apoptotic feature, in the scattered granulosa cells, but cumulus cells with normal ultrastructure were also seen in the same follicles [7]. The activity of neutral $\mathrm{Ca}^{2+} / \mathrm{Mg}^{2+}-$ dependent endonuclease was detected in granulosa cells but not in cumulus cells of atretic follicles [8]. These findings confirm that apoptosis occurs in granulosa cells, but not cumulus cells, in the atretic Graafian follicles of porcine ovaries [4-7].

Interestingly, in porcine follicles of the early stage of atresia, apoptotic granulosa cells, apoptotic 
bodies and cell debris were phagocytosed by neighboring granulosa cells with normal ultrastructure [7]. Positive staining of Sambucus sieboldiana agglutinin (SSA), a specific lectin for Sia $2,6 \mathrm{Gal} / \mathrm{GalNAc}$ structure, was shown in the granulosa cells of atretic follicles, but no positive staining was seen in those of healthy follicles [9]. The analysis of the backbone structures of SSApositive glycans revealed that glycoprotein on granulosa cells of atretic follicles had Sia $2,6 \mathrm{Gal} \beta 1,4 \mathrm{GlcNAc}$ on complex-type N-glycans, suggesting that sialyltransferase ST6Gal I, a $\alpha 2,6-$ sialyltransferase, increases in granulosa cells during follicular atresia [10]. Moreover, reverse transcription-polymerase chain reaction (RT-PCR) analysis for ST6Gal I mRNA confirmed it was up regulated in granulosa cells of atretic follicles, indicating that the alteration of glyco-conjugates is caused by ST6Gal I in granulosa cells during atresia [10]. The phagocytosis by neighboring healthy granulosa cells may be caused by changes of cell membrane glyco-conjugates in the granulosa cells during atresia. The regulation mechanisms of glyco-conjugate alteration and of phagocytosis in neighboring granulosa cells are not well understood.

We previously produced two unique monoclonal antibodies, PFG-5 and PFG-6 antibodies for examing the molecular mechanisms regulating granulosa cell apoptosis in porcine follicles: IgM monoclonal antibody, PFG-5, is capable of inducing granulosa cell apoptosis, and IgG monoclonal antibody, PFG-6, is not capable of inducing granulosa cell apoptosis and inhibits IgM antibodyinduced apoptosis [11]. PFG-5 and PFG-6 were produced against granulosa cells prepared from healthy antral follicles of porcine ovaries. These monoclonal antibodies are believed to recognize a novel cell-death receptor, a member of the tumor necrosis factor-receptor superfamily, and a decoyreceptor involved in regulation of granulosa cell survival and death, but detailed mechanisms of the regulation of the novel cell-death receptor system are not well understood.

In the present study, to examine the molecular mechanisms regulating apoptosis and phagocytosis in granulosa cells of porcine follicles, we produced a monoclonal antibody against granulosa cells prepared from atretic follicles.

\section{Materials and Methods}

\section{Preparation of monoclonal antibody against follicular granulosa cells}

We produced a monoclonal antibody against porcine granulosa cells as described previously [11, 12]. Briefly, preovulatory antral follicles, $3-5 \mathrm{~mm}$ in diameter, were dissected from ovaries obtained from mature sows at a local slaughterhouse. They were classified as morphologically healthy or atretic, and then subdivided into early and progressed atretic follicles based on the progesterone/estradiol-17 $\beta$ ratio in follicular fluid assessed by $\left[{ }^{125} \mathrm{I}\right]$-radioimmunoassay (Bio-Mérieux, Marcyl'Etolle, France) [4]. When the progesterone/ estradiol- $17 \beta$ ratio was in the range 15 to 20 , the follicle was classified as early atresia. Granulosa cells were isolated, and the cells with viability of more than $95 \%$ were used as antigen for immunization.

Female BALB/c mice ( 8 weeks old) were purchased from Japan Clea (Tokyo, Japan) and received humane care as outlined in the "Guide for the Care and Use of Laboratory Animals" (Kyoto University Animal Care Committee according to NIH \#86-23; revised 1999). They were immunized intravenously with isolated granulosa cells $\left(10^{6}\right.$ cells/mouse, bi-weekly). After assessment of antibody production estimated by immunofluorescence staining, the spleen cells prepared from immunized mice were fused with Sp2/OAg14 mouse myeloma cells by standard hybridization techniques using polyethylene glycol 1500 (Boehringer Mannheim, Indianapolis, IN, USA). After selection of the hybridoma cells producing IgM antibody against granulosa cells screened by immunofluorescence staining, one hybridoma cell line was cloned by limiting dilution and named PAG-1 clone. Then, female BALB/c mice ( 9 weeks old), which were pretreated with an intraperitoneal injection of $2,6,10,14$ tetramethylpentadecane $(0.5 \mathrm{ml} /$ mouse; Sigma) 5 weeks before, intraperitoneally received PAG-1 hybridoma cells $\left(1 \times 10^{7}\right.$ cells/mouse $)$. Two weeks after hybridoma injection, ascites were obtained from the mice. The antibody was purified using a hydroxyapatite-column (Asahi Optical, Tokyo, Japan) for preparative HPLC system (Amersham Pharmacia Biotech, Piscataway, NJ, USA), concentrated with an ultrafiltration membrane (XM50; Amicon, Beverly, MA, USA), heat- 
inactivated at $56 \mathrm{C}$ for $45 \mathrm{~min}$, and sterilized by filtration through $0.22-\mu \mathrm{m}$ porefilters (Millipore, Marlborough, MA, USA). The protein concentration was determined by measurement of the optical density at $280 \mathrm{~nm}$.

\section{Immunofluorescence staining}

Immunofluorescence staining was performed as described previously $[11,12]$. Briefly, serial frozen sections ( $5 \mu \mathrm{m}$ thick) of porcine ovaries were fixed with -80 C-acetone for $5 \mathrm{~min}$, preincubated with $1 \%$ normal goat serum (Sigma) diluted with phosphate buffered saline containing $1 \%$ bovine serum albumin (PBS-BSA), and incubated with immunized mouse serum (1/10 dilution with PBSBSA), hybridoma culture supernatant $(1 / 100$ dilution with PBS-BSA) or purified monoclonal antibody $(0.01 \mu \mathrm{g} / \mathrm{ml}$ dilution with PBS-BSA) for 18 $\mathrm{h}$ at $4 \mathrm{C}$. The sections were incubated with fluorescein isothiocyanate (FITC)-conjugated goat anti-mouse IgM antibody (1/400 dilution with PBSBSA; American Qualex, La Mirada, CA, USA) for 2 $h$ at RT, and then examined with a fluorescence microscope (BX51-fluoro system, Olympus, Tokyo, Japan) or a confocal laser scanning microscope (Fluoroview FV3000, Olympus). As negative controls, adjoining sections were incubated with diluted non-immunized mouse serum (1/10 dilution with PBS-BSA) or mouse IgM $(0.1 \mu \mathrm{g} / \mathrm{ml}$ in PBS-BSA; Sigma).

\section{Western blotting analysis}

As described previously [11, 12], homogenized samples ( $20 \mu \mathrm{g} /$ lane) of the cell membrane fraction prepared from granulosa cells of healthy, early atretic and progressed atretic follicles, luteal body, oviduct, uterus, testis, liver, kidney, adrenal gland, pancreas, stomach, small intestine, large intestine, spleen, thymus, brain, heart, lung and skeletal muscle were separated by sodium dodecyl sulfate$4 \%$ polyacrylamide gel electrophoresis (SDSPAGE). The protein fraction was transferred onto nitrocellulose membranes (Amersham Pharmacia). The membranes were incubated with diluted PAG1 antibody $(0.01 \mu \mathrm{g} / \mathrm{ml})$ for $18 \mathrm{~h}$ at $4 \mathrm{C}$, incubated with horseradish peroxidase-conjugated goat antimouse IgM antibody (Jackson Immunoresearch Laboratories, West Grove, PA, USA) for $2 \mathrm{~h}$ at RT, and then chemiluminescence was visualized using an ECL system (Amersham Pharmacia) according to the manufacturer's protocol. The chemilumi- nescence was recorded with a digital recorder (LAS-1000, Fuji Film, Tokyo, Japan), and the protein expression levels were quantified using ImageGauge software (Fuji Film) on a Macintosh computer.

\section{Glycosidase treatment}

We performed glycosidase treatment as described previously [10]. Briefly, the cell membrane preparations of granulosa cells of healthy, early atretic and progressed atretic follicles were digested with $0.8 \mathrm{U} / \mathrm{ml}$ ureafaciens sialidase (Nacalai Tesque, Kyoto, Japan) in $0.1 \mathrm{M}$ sodium acetate buffer ( $\mathrm{pH} 5.0$ ), which cleaves sialic acid linkage, for $1 \mathrm{~h}$ at $37 \mathrm{C}$, and then treated with $10 \mathrm{U} /$ $\mathrm{ml}$ of $\mathrm{N}$-glycosidase $\mathrm{F}$ (Boehringer Mannheim) in $0.1 \mathrm{M}$ sodium phosphate buffer ( $\mathrm{pH}$ 7.3) containing $25 \mathrm{mM}$ ethylenediaminetetraacetic acid disodium salt and $1 \%$ Triton $\mathrm{X}-100$, which cleaves aspartylglycosylamine linkages, for $1 \mathrm{~h}$ at $37 \mathrm{C}$. Digested preparations were analyzed by Western blotting as described above.

\section{Statistical analysis}

ANOVA for biochemical data was carried out using StatView-IV on a Macintosh computer. Differences of $\mathrm{P}<0.05$ were considered significant.

\section{Results}

\section{Characterization of antigens by Western blotting and glycosidase treatment}

The antigens on granulosa cells of healthy, early atretic and progressed atretic follicles were characterized by conventional Western blotting. In the homogenized samples of granulosa cell membrane prepared from early atretic and progressed atretic follicles, two specific bands with molecular weights of 38 and $64 \mathrm{kD}$ were observed on nitrocellulose filters treated with PAG-1 antibody (Fig. 1A; lane 2 and 3, respectively). In the homogenized samples of granulosa cell membrane prepared from healthy follicles, 2 bands with molecular weights of 38 and $42 \mathrm{kD}$ were observed (Fig. 1A; lane 1). During follicular atresia, the 64$\mathrm{kD}$ band protein increased (Fig. 1B). No immunopositive reaction against preparations of luteal body, oviduct, uterus, testis, liver, kidney, adrenal gland, pancreas, stomach, small intestine, large intestine, spleen, thymus, brain, heart, lung or 

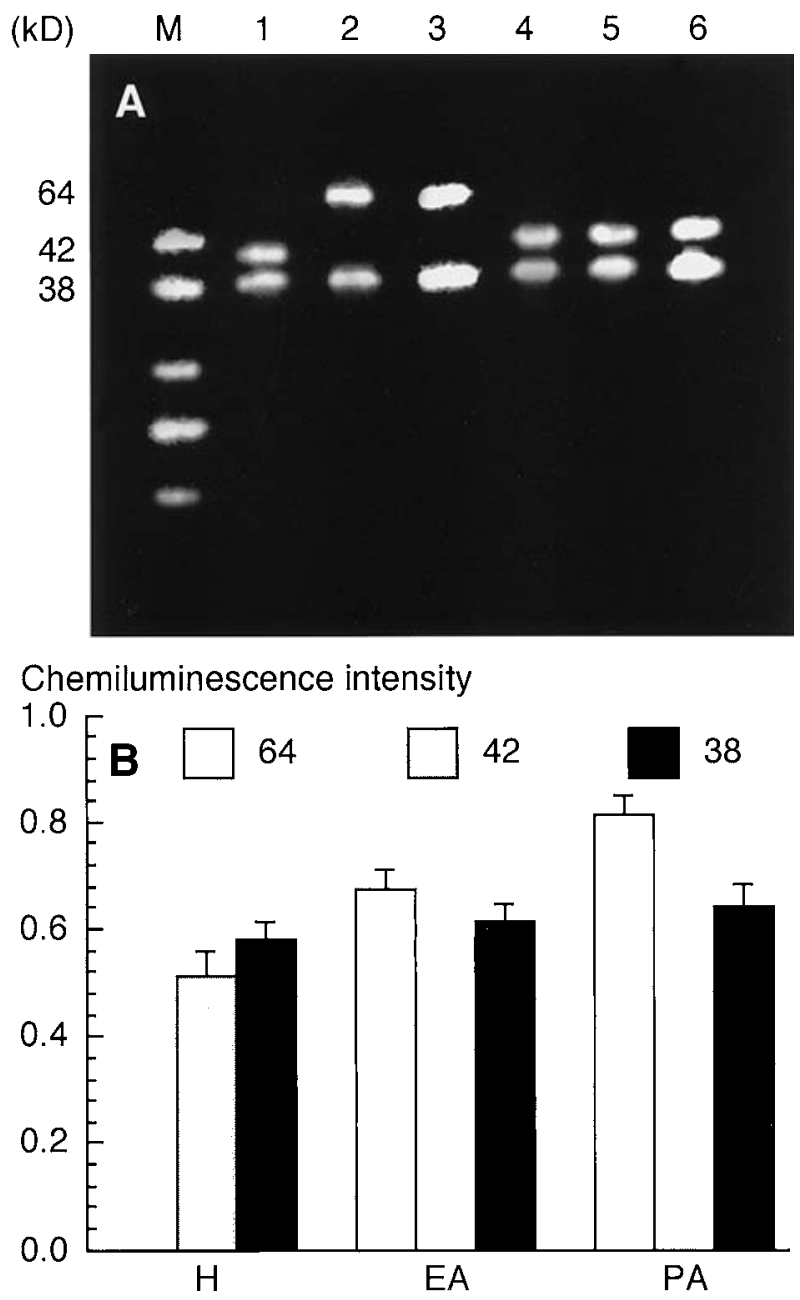

Fig. 1. Representative results of Western blotting analysis of granulosa cell membrane antigens recognized by PAG-1 (A) and the results of semiquantitative analysis for the immunoreaction (B) are shown. Cell membrane fractions prepared from granulosa cells of healthy, early atretic and progressed atretic follicles were separated by SDS-PAGE (lane 1, 2 and 3, respectively). The samples from healthy, early atretic and progressed atretic follicles were treated with glycosidase, and then separated by SDS-PAGE for Western blotting analysis (lane 4,5 and 6, respectively).

skeletal muscle was seen (data not shown).

After glycosidase treatment, the 64-kD band, which was detected in the granulosa cells of early atretic and progressed atretic follicles, shifted to 42 kD (Fig. 1A; lane 5 and 6, respectively). No change was seen in $38-$ or $42-\mathrm{kD}$ bands (Fig. 1A; lane 4,5 and 6).
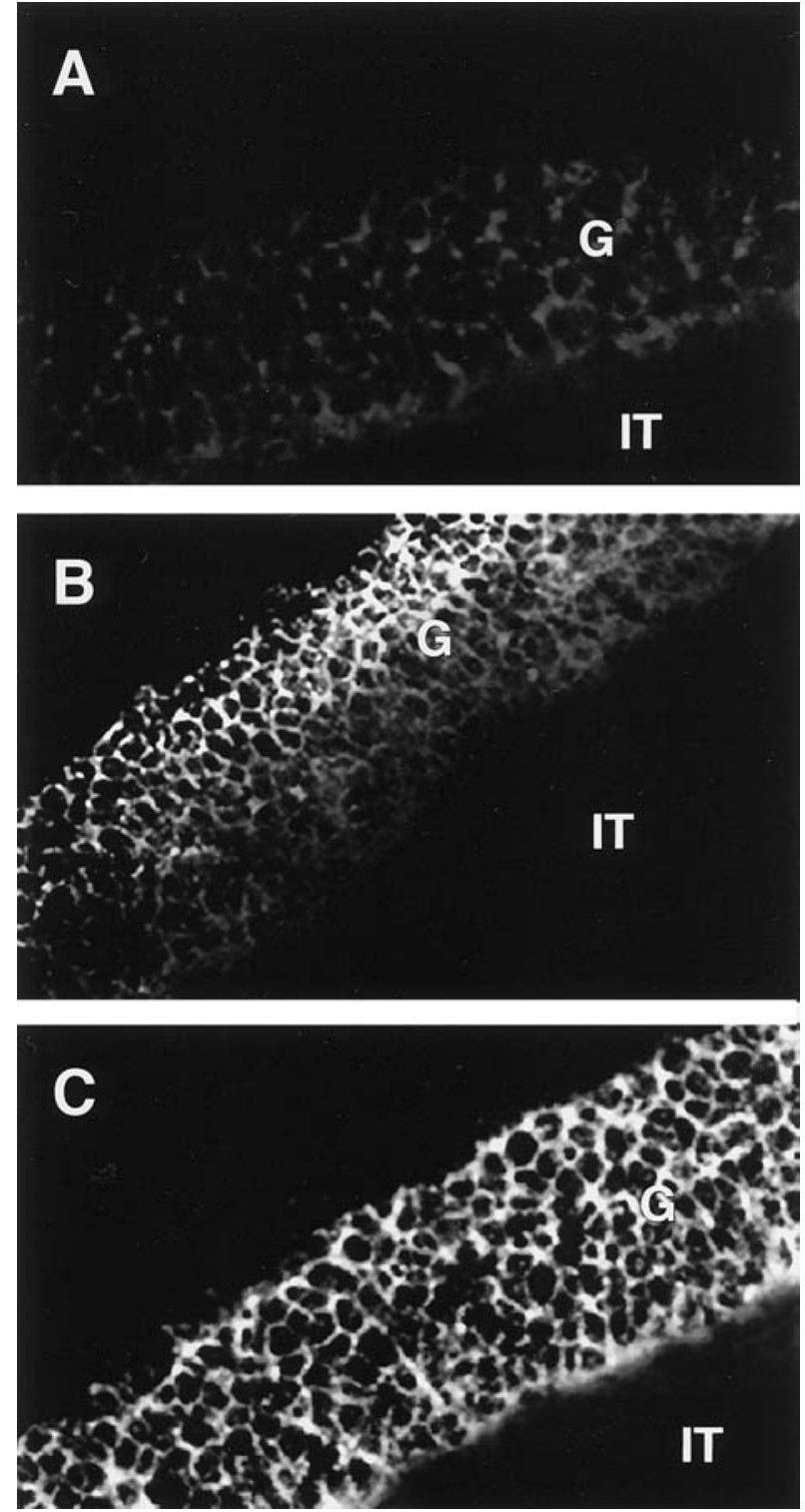

Fig. 2. Composite confocal images of frozen sections of healthy, early atretic and progressed atretic follicles (lane A, B and C, respectively). The sections were stained with PAG-1 antibody and with FITCconjugated anti-mouse IgM, to show the distribution of PAG-1 antigens. The follicles were optically sectioned at $0.5 \mu \mathrm{m}$ and five serial images were generated using the confocal microscope. G: granulosa cell layer, IT: internal theca layer. $\times 200$.

\section{Immunohistochemical characterization}

Immunofluorescence analysis on ovarian sections revealed that PAG-1 antibody strongly reacted with granulosa cells of early atretic and progressed atretic follicles (Fig. 2B and C, respectively), especially with the inner wall of 
granulosa cell layers of early atretic follicles (Fig. 2B). Weak fluorescent staining was seen in the granulosa cell layer of healthy follicles (Fig. 2A). No immuno-positive staining was detected in internal or external theca layers, basement membrane of follicles or ovarian stroma cells (Fig. $2 \mathrm{~A}, \mathrm{~B}$ and $\mathrm{C}$ ).

\section{Discussion}

In the present study, to examine the molecular mechanisms of the post-apoptotic process in granulosa cells, we used granulosa cells prepared from atretic follicles as the antigen, produced hybridoma cells, and selected a hybridoma clone. The clone, named as PAG-1, produced monoclonal IgM antibodies against cell-membrane proteins of granulosa cells. PAG-1 antibody was not capable of inducing granulosa cell apoptosis (preliminary experiment). Immunohistochemical staining and conventional Western blotting analysis revealed that the PAG-1 antibody showed specific binding to granulosa cells of porcine ovarian follicles, but no specific binding to cumulus cells, oocytes, internal or external theca cells. Moreover, this antibody had no binding to the luteal body, oviduct, uterus, testis, liver, kidney, adrenal gland, pancreas, stomach, small intestine, large intestine, spleen, thymus, brain, heart, lung, adrenal gland, thyroid gland or skeletal muscle. Two immunopositive protein bands ( 38 and $64 \mathrm{kD}$ ) recognized by PAG-1 antibody were demonstrated in granulosa cell membrane samples prepared from early atretic and progressed atretic follicles, and the $64-\mathrm{kD}$ band increased during follicular atresia. In the samples from healthy follicles, 38- and 42-kD bands were observed. Interestingly, the $64-\mathrm{kD}$ band shifted to $42 \mathrm{kD}$ after glycosidase treatment, but no change was seen in the 38- or $42-\mathrm{kD}$ bands. Thus, the 64$\mathrm{kD}$ band is glycoconjugated protein, and the 38and $42-\mathrm{kD}$ bands are proteins without glycoconjugation.

As we previously reported $[9,10]$, SSA-lectin histochemistry and blot analysis showed two specific bands (62 and $64 \mathrm{kDa}$ ), which were recognized by SSA and observed only in granulosa cells of early atretic and progressed atretic follicles, and these SSA-positive bands completely disappeared after glycosidase digestion as in the present study. Moreover, these glycoproteins increased during follicular atresia. We presume that the $64-\mathrm{kD}$ band glycoprotein detected by SSAlectin blotting is the same membrane protein as the 64-kD band recognized by PAG-1 antibody. The glycostructure of the $64-\mathrm{kD}$ glycoprotein is considered to be Sia $\alpha 2,6 \mathrm{Gal} \beta 1,4 \mathrm{GlcNAc}$ on complex-type $\mathrm{N}$-glycans, suggesting that only ST6Gal I of the four distinct $\alpha 2,6$-sialyltransferases catalyzes $\alpha 2,6$-sialic acid transfer in the majority of the increased glycoproteins of granulosa cells during follicular atresia. The $42-\mathrm{kD}$ band protein may be glucoconjugated by the enzyme.

On the basis of our previous [4-10] and present data, we hypothesize as below. The structure of the granulosa cell layer is well kept in healthy follicles, and granulosa cells proliferate and produce many growth factors and hormones to grow and mature the oocytes. In follicles of the early stage of atresia, apoptosis occurs in scattered granulosa cells located on the inner surface of the follicular wall. In granulosa cells undergoing apoptosis, the inducing signal, $\alpha 2,6$-sialyltransferase, ST6Gal-I, is transcribed and translated, and then this enzyme catalyzes the cell membrane protein $(42-\mathrm{kD}$ protein). The glycoconjugated cell membrane protein (64-kD glycoprotein) acts as a trigger for phagocytosis. Thus, apoptotic granulosa cells, apoptotic bodies and cell debris are phagocytosed by neighboring granulosa cells with normal ultrastructure. In our laboratory, PAG-1 antibody has been used to screen for the phagocytosis receptor on the granulosa cell membrane, which acts as a key factor in scavenging the degenerative cells by normal cells. The antibody will be a useful and sensitive probe for investigating the regulation mechanism of phagocytosis, for elucidating cell surface mechanisms for the regulation of the scavenging function, and for defining the intercellular pathway of the scavenging signal transduction in granulosa cells of porcine ovaries.

\section{Acknowledgements}

This work was supported by a Grant-in-Aid for Creative Scientific Research (13GS0008) to N. M. from the Ministry of Education, Culture, Sports, Science and Technology, Japan. We are grateful to Dr. Seishiroh Katoh (Kobe University, Kobe, Japan) for advice on the preparation of granulosa cells and the determination of healthy and atretic follicles. 


\section{References}

1. Hirshfield AN. Development of follicles in the mammalian ovary. Int Rev Cytol 1991; 124: 43-101.

2. Tilly JL. Cell death and species propagation: molecular and genetic aspects of apoptosis in the vertebrate female gonad. In: Lockshin RA, Zakeri Z, Tilly JL (eds.), When Cells Die. New York, WileyLiss; 1998 ; 431-452.

3. Manabe N, Kimura Y, Myoumoto A, Matsushita $H$, Tajima C, Sugimoto M, Miyamoto H. Role of granulosa cell apoptosis in ovarian follicle atresia. In: Yamada T, Hashimoyto Y (eds.), Apoptosis: Its Roles and Mechanism. Tokyo, Academic Societies Book Press, 1998; 97-111.

4. Manabe N, Imai $\mathbf{Y}$, Ohno H, Takahagi $\mathbf{Y}$, Sugimoto $\mathbf{M}$, Miyamoto $\mathbf{H}$. Apoptosis occurs in granulosa cells but not cumulus cells in the atretic antral follicles in the pig ovaries. Experientia 1996; 52: 647651.

5. Manabe N, Myoumoto A, Kimura Y, Imai $\mathbf{Y}$, Sugimoto M, Sakamaki K, Okamura Y, Fukumoto M, Miyamoto H. Regulatory mechanisms of granulosa cell apoptosis in porcine ovarian follicle atresia. In: Miyamoto H, Manabe N. (eds.), Reproductive Biology Update. Kyoto, Shokado Press, 1998; 23-35.

6. Nakayama M, Manabe N, Nishihara S, Miyamoto H. Species-specific differences in apoptotic cell localization in granulosa and internal theca cells during follicular atresia in porcine and bovine ovaries. J Reprod Dev 2000; 46: 147-156.

7. Sugimoto M, Manabe N, Kimura Y, Myomoto A, Imai $\mathbf{Y}$, Ohno $\mathbf{H}$, Miyamoto $\mathbf{H}$. Ultrastructural changes in granulosa cells in porcine antral follicles undergoing atresia indicate apoptotic cell death. J
Reprod Dev 1998; 44: 7-14.

8. Manabe N, Imai $\mathbf{Y}$, Kimura $\mathbf{Y}$, Myoumoto A, Sugimoto M, Miyamoto H, Okamura Y, Fukumoto M. $\mathrm{Ca}^{2+} / \mathrm{Mg}^{2+}$-Dependent endonuclease but not $\mathrm{Ca}^{2+}$-Dependent, $\mathrm{Mg}^{2+}$-Dependent or cationindependent endonuclease is involved in granulosa cell apoptosis of pig atretic follicles. I Reprod Dev 1996; 42: 247-253.

9. Kimura Y, Manabe N, Matsushita H, Tajima C, Myoumoto A, Miyamoto $\mathbf{H}$. Examination of granulosa cell glycoconjugates which change during follicular atresia in the pig ovary. J Reprod Dev 1998; 44: 35-44.

10. Kimura $\mathbf{Y}$, Manabe N, Nishihara S, Matsushita $\mathbf{H}$, Tajima C, Wada S, Miyamoto H. Up-regulation of the $\alpha 2,6-$ sialyltransferase messenger ribonucleic acid increases glycoconjugates containing $\alpha 2,6-$ linked sialic acid residues in granulosa cells during follicular atresia of porcine ovaries. Biol Reprod 1999; 60: 1475-1482.

11. Manabe N, Myoumoto A, Tajima C, Fukumoto M, Nakayama M, Uchio K, Yamaguchi M, Miyamoto H. Immunochemical characteristics of a novel cell death receptor and a decoy receptor on granulosa cells of porcine ovarian follicles. Cytotechnology 2000; 33: 189-201.

12. Myoumoto A, Manabe N, Imai $Y$, Kimura $Y$, Sugimoto M, Okamura Y, Fukumoto M, Sakamaki K, Yonehara S, Niwano $Y$, Miyamoto $H$. Monoclonal antibodies against pig ovarian follicular granulosa cells induce apoptotic cell death in cultured granulosa cells. J Vet Med Sci 1997; 59: 641649. 